\title{
Quality of life
}

science: Implications for cancer nursing

By Sally E. Thorne and Carol R. Jillings

\begin{abstract}
Recent developments in the sophistication of health-related quality of life measurement instruments mean that quality of life assessment can now be integrated into many components of cancer treatment and program evaluation as well as in clinical trials of new therapeutic approaches. While such initiatives clearly underscore a welcomed enthusiasm for attending to the effects of illness and treatment upon human lived experience, uncritical acceptance of their application may create new problems for oncology mursing researchers and clinicians. In this paper, a critical analysis of quality of life science makes explicit both its strengths and its limitations, and challenges oncology murses to apply reasoned judgment to the acceptance of appropriate applications in both clinical research and practice.
\end{abstract}

The notion of quality of life pervades the literature concerned with psychosocial aspects of illness and the outcomes of treatment (Farquhar, 1995). Quality of life (QoL) is particularly relevant to all spheres of cancer nursing, especially clinical practice and research, where practitioners and scientists alike struggle to achieve optimal outcomes for persons with cancer and their families. As the general knowledge of QoL grows, various definitions proliferate in an attempt
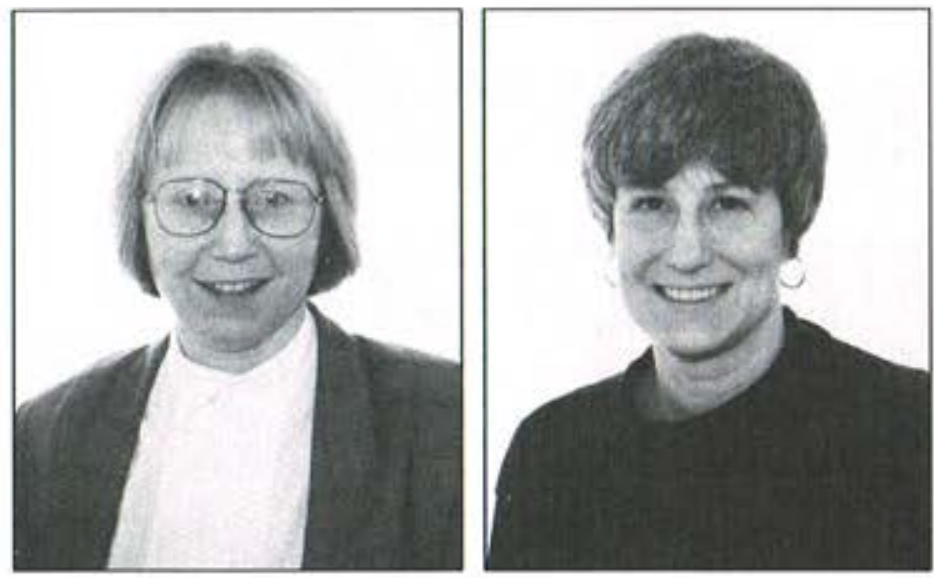

Sally Thorne

Carol Jillings to describe the concept, its characteristics and, if possible, its parameters. Both objective and subjective approaches to defining QoL have emerged from clinical practice and research (Rosenberg, 1995). The former have comprised attempts to develop indices or measurements of QoL, often linked to the individual patient's physiological status and proximity to remission or cure. Subjective approaches to description of QoL have taken the focus toward documentation of lived experiences with illness, including patient definitions of significant aspects of QoL.

While objective and subjective studies of QoL need not be mutually exclusive, the efficiency afforded by objective measurement creates the potential for objectification of the concept. This issue has particular significance in cancer nursing because of the complexity of the illness experience from diagnosis, through treatment, and toward rehabilitation or palliation. When one conceptualizes cancer as a chronic illness, the attendant issues of client/family response, illness management, and illness trajectory (Strauss, Corbin, Fagerhaugh, Glaser, Maines, Suczek \& Weiner, 1984) become relevant templates through which QoL can be considered. When factored into clinical practice and research, these dimensions of long-term illness render QoL more complex than standardized measures or norms might suggest. Subjective factors arising from patients' illness experiences - individual beliefs, coping styles, strengths, choices - cannot be brushed aside nor objectively assessed as components of functional status or symptomatology. Thus, when we reframe QoL in the context of cancer illness experience, the evolving science and the research-generated knowledge that guides our practice requires a shift in focus.

\section{Quality of life measurement}

Cancer clinical practice and science have been dominated by an entirely curative focus for several generations. Over the past decade or so, nursing has been influential in stimulating recent serious attention to quality of life as an equally important outcome measure in the lives of those afflicted (Herold \& Roetzheim, 1992; Hunt, 1993). Considerable research into measurement tools shows that one can document significant distinctions between the two outcomes. For example, breast cancer survivorship literature reveals that cancer and

\section{ABRÉGÉ}

\section{LA SCIENCE DE LA QUALITÉ DE VIE: IMPLICATIONS SUR LES SOINS INFIRMIERS EN ONCOLOGIE}

Les récents raffinements apportés aux instruments de mesure de la qualité de vie dans le contexte des soins de santé signifient que l'on peut désormais intégrer l'évaluation de la qualité de vie à de nombreuses composantes du traitement du cancer et de l'évaluation des programmes ainsi qu'aux essais cliniques de nouvelles méthodes thérapeutiques. Ėtant donné que de telles initiatives sont nées d'un réel intérêt de connaître les effets de la maladie et du traitement sur le vécu humain, une acceptation non critique de leur application pourrait créer de nouveaux problèmes pour les chercheures et les cliniciennes oeuvrant dans le domaine des soins infirmiers en oncologie. Cet article présente une analyse critique de la science de la qualité de vie et en démontre les points forts ainsi que les limitations; il invite les infirmières en oncologie à appliquer jugement et pondération afin de sélectionner les applications appropriées aux domaines de la recherche et de la pratique cliniques.

Sally E. Thorne, RN, PhD, and Carol R. Jillings, RN, PhD, are both associate professors at the University of British Columbia School of Nursing, Vancouver, British Columbia. 
its treatment (especially mastectomy) can have a negative quality of life impact for decades after cure is pronounced (Ferrans, 1994; Ferrell, Dow, Leigh, Ly \& Guladekaram, 1995; Wyatt, Kurtz \& Liken, 1993). In contrast, the care and treatment of a predominantly terminal disease such as primary lung cancer can create a demonstrable improvement for the quality of remaining life without influencing mortality (Schmitt, 1993). Several factors, including consumer pressure, the toxicity of some cancer treatments, and the stabilization or decline of major improvements in cancer cure rates have influenced many scientists to argue for widespread inclusion of QoL measures in clinical trials of various cancer treatments (Osoba, 1994).

As QoL measurement has increased in popularity, debate as to the validity, reliability, sensitivity and specificity of various instruments has occupied considerable attention in the literature (Bowling, 1995; Donovan, Sanson-Fisher \& Redman, 1989; Rosenberg, 1995). The argument has been made that the initial problems associated with standardized measurement of this subjective construct are now resolved, and that the major impediment to its widespread application is the reluctance of research scientists to include it in their designs (Osoba, 1994). Since it is almost universally established that self-report is the only valid source of QoL data, patient compliance in completing instruments is required. Further, since most researchers believe that the phenomenon is multifaceted (such as the physical, psychological and interpersonal domains articulated by Padilla, Ferrell, Grant \& Rhiner, 1990), the tools tend to be cumbersome and time-consuming. However, a recent body of literature has emerged helping scientists work out the details of building QoL measurement into their clinical trials (Hayden et al., 1993; Jalowiec, 1990).

\section{Interpretations of quality of life data}

Despite the impressive movement in this direction (from cure alone and toward a more humanistic orientation), several serious problems may arise from the illusion that the puzzles of QoL measurement have now been mastered. If we determine, for example, that two treatment protocols have similar cure rates but very different quality of life impacts, the results of QoL research will be helpful in providing us with useful information upon which to guide important clinical decisions and encourage informed consent on the part of patients. However, this new information will be based upon generalized population data (the law of averages), and may therefore make individual variations even more difficult to appreciate.

The case of breast cancer illustrates the problem that may arise from conflating population data with individual experience. While it is undoubtedly true that unnecessary mastectomy has jeopardized the quality of life of many women (Henderson, 1995), there will still be women whose concern about recurrence will lead them to demand prophylactic double mastectomy over a simple lumpectomy regardless of the mortality statistics. In other words, the context of each individual illness experience often makes all of the best intended population data meaningless in its application to a single case (Greer, 1987). In fact, the more success we have with establishing population norms that summarize generalizations about quality of life, the more we stand to lose touch with that very quality that made it so challenging to measure - the individual subjective nature of the construct. Since such measures provide such useful information about population data, it is easy to forget that they actually tell us nothing about any individual case.

In times of extreme pressure upon the health care system for fiscal restraint and resource allocation (Yasko \& Fleck, 1984/1995), it is naive to think that QoL data will not be applied to a defence of health care policy decisions (Gioiella, 1995). So, for example, if the cost of the prophylactic mastectomy exceeds that of the standard lumpectomy, how long will it be before health insurance schemes deny the additional expenses and cite QoL research as their justification? In fact, as Katz (1995) has pointed out, there is a strong consensus among quality of life researchers that the primary importance of QoL research lies in its application to program planning and policy making.

\section{The chronicity factor in cancer illness}

While some important advantages of QoL research can easily be demonstrated in short-term applications such as treatment protocol side effects, cancer is rapidly becoming a chronic illness (Dorsett, 1991). Examination of some of the distinctions between acute and chronic illness experiences illuminates another facet of the difficulties associated with QoL science. Acute illness typically heralds dramatic and predictable changes in quality of life. Our priorities the day after a massive myocardial infarction or a traumatic injury will inevitably be different than they were the day before. Because such distinctions are easy to quantify, these kinds of quality of life changes are amenable to translation into the kinds of arguments that justify expenditures for health care services. In the case of chronic disease, however, illness experience is typically characterized by coping, gradual adaptation, learning to live differently, and finding appropriate resources for managing the implications of illness. The objective of the individual with chronic illness is typically to sustain as normal a life as possible and to maintain as high a quality of life as possible (sometimes against rather overwhelming odds). The spectacular creativity of the human spirit means that many chronically ill individuals are capable of orchestrating their lives in such a manner that they maintain lives of high quality. In such cases, quantification of QoL could lead to the damaging conclusion that the impact of the disease on quality of life is minimal and, therefore, that the chronically ill require little social commitment in the way of resource allocation.

Thus, when we examine the logic of QoL science from the chronic illness perspective, we are inevitably challenged with the fact that population-based norms and individual cases may bear little relationship to one another, and therefore that the science has the potential to mislead clinical practice (Greer, 1987). The chronic illness world reveals countless individuals who maintain a high quality of life in the face of unbelievably toxic symptoms, and also an equally impressive group of individuals who have an extremely impaired quality of life without tangible criteria (the "worried well", for example). And while we often try to understand what it is that distinguishes those who live well from those who cannot despite apparently similar disease conditions, correlations are inconsistent and interpretations are often misleading. Nurses recognize that all chronic illness has the potential for major and minor influences on quality of life throughout the course of the disease and that a chronically ill person's quality of life will change in response to a range of disease and context-related phenomena (Hunt, 1993; Lindley \& Hirsch, 1994). The "art" of nursing toward quality of life is therefore almost totally divorced from the science of learning about population patterns. As the science becomes more convincing, will we lose the ability to appreciate the inherent value of the art?

\section{Implications for oncology nursing research}

Identification of ways in which statistical measures of QoL can be misleading, and awareness of what it means when an illness has become chronic, lead us toward the realization that a cautious approach to QoL research is required (Mast, 1995). There may be some research questions that ought not to be asked, or ought to be asked quite differently. Our common sense tells us that we would have much different feelings and experiences following a harrowing diagnostic test, during a period of waiting for treatment or on the event of being given a poor prognosis. What would it mean, therefore, if the research showed no measurable QoL change? For example, Zacharias, Gilg \& 
Foxall (1994) report that patients with gynecological cancer and their spouses are relatively satisfied with their quality of life. Does this mean that gynecological cancer is not a sufficiently dramatic event to influence life's quality? Or, alternatively, does it mean that excellent care and counselling may have alleviated many of the noxious effects of anxiety and treatment? One can easily see that different interpretations of such findings could have major implications for psychosocial service protocols, care priorities and staff resource allocation within a cancer facility.

Although it can help us to understand population norms, a statistical measure of quality of life cannot truly tell us about how individuals experience cancer (Mast, 1995; Philips, 1995). Among those important questions that QoL measures cannot hope to answer are: What is it that allows some people to transcend the impact of pain, fear and disfigurement and experience a high quality of life in cancer? How do individuals come to know (and communicate to us) the unique qualities that will determine how they can best proceed through a cancer illness? What cultural variations are there within the construct that we call quality of life? And how is it that the human spirit attaches meaning to illness? Such questions properly belong within the domain of qualitative and philosophical inquiry within nursing, and reduction to quantifiable variables will inevitably distort our understanding of such issues. At the same time, qualitative and philosophical inquiries alone are unlikely to have persuasiveness in the health policy arena and therefore to effect widespread pragmatic change in the organization of nursing and health care services (Mast, 1995).

\section{References}

Benner, P. (1985). Quality of life: A phenomenological perspective on explanation, prediction, and understanding in human science. Advances in Nursing Science, 8(1), 1-14.

Bowling, A. (1995). What things are important in people's lives?: A survey of the public's judgments to inform scales of health related quality of life. Social Science \& Medicine, 41(10), 1447-1462.

Donovan, K., Sanson-Fisher, R.W., \& Redman, S. (1989). Measuring quality of life in cancer patients. Journal of Clinical Oncology, $7(7), 959-968$.

Dorsett, D.S. (1991). The trajectory of cancer recovery. Scholarly Inquiry for Nursing Practice, 5(3), 175-184.

Farquhar, M. (1995). Definitions of quality of life: A taxomony. Journal of Advanced Nursing, 22, 502-508.

Ferrans, C.E. (1994). Quality of life through the eyes of survivors of breast cancer. Oncology Nursing Forum, 21(10), 1645-1651.

Ferrell, B.R., Dow, K.H., Leigh, S., Ly, J. \& Gulasekaram, P. (1995). Quality of life in long-term cancer survivors. Oncology Nursing Forum, 22(6), 915-922.

Gioiella, E.C. (1995). Quality of life revisited. Nursing Science Quarterly, 8(3), 97.

Greer, D.S. (1987). Quality of life measurement in the clinical realm. Journal of Chronic Diseases, 40(6), 629-630.

Hayden, K.A., Moinpour, C.M., Metch, B., Feigl, P., O'Bryan, R.M., Green, S. \& Osborne, C.K. (1993). Pitfalls in quality-of-life assessment: Lessons from a Southwest oncology group breast cancer clinical trial. Oncology Nursing Forum, 20(9), $1415-1419$.

Henderson, I.C. (1995). Paradigmatic shifts in the management of breast cancer. New England Journal of Medicine, 332(14), 951-952.

Herold, A.H. \& Roetzheim, R.G. (1992). Cancer survivors. Primary Care: Clinics in Office Practice, 19(4), 779-791.

Hunt, J. (1993). Quality of the patient's life versus the quest for a cure. British Journal of Nursing, 2(16), 819-822.

Jalowiec, A. (1990). Issues in using multiple measures of quality of life. Seminars in Oncology Nursing, 6(4), 271-277.
Quality of life research clearly represents a trend toward valuing human subjective experience and factoring it into health care decision-making. As such, it can serve as a valuable tool for nurses in their clinical research. However, critical analysis of the implications of the fruits of this research make it apparent that quality of life findings might well be applied in ways that are counter-intuitive to the direction that cancer nurses recognize as appropriate. Statistical norms about quality of life must never take precedence over the conclusions that nurses can draw about individuals on the basis of a thoughtful and systematic assessment of what gives meaning to their cancer experience. While they can add to our understanding, scores on a scale cannot match the depth of knowledge that derives from assessment in the context of ongoing health care relationships. And ease of measurement ought not to shift our research priorities away from honouring the mysterious capacities of individuals and toward documentation of population commonalities (Benner, 1985; Ketefian, 1995).

The state of the art in QoL research presents us with challenges that surface in areas as diverse as ethics, health policy, and practice standards. By grounding ourselves in the central purpose of our clinical and research activities, and remembering that our ultimate purpose is the service of very real people, we can approach new problems and questions with confidence and courage.

Katz, S. (1987). The science of quality of life. Journal of Chronic Diseases, 40(6), 459-463.

Ketefian, S. (1995). Individual versus community: Ethical issues in quality of life. Nursing Science Quarterly, 8(3), 98-99.

Lindley, C.M. \& Hirsch, J.D. (1994). Oncology nurses' attitudes, perceptions, and knowledge of quality-of-life assessment in patients with cancer. Oncology Nursing Forum, 21(1), 103-108.

Mast, M.E. (1995). Definition and measurement of quality of life in oncology nursing research: Review and theoretical implications. Oncology Nursing Forum, 22(6), 957-964.

Padilla, G.V., Ferrell, B., Grant, M.M. \& Rhiner, M. (1990). Defining the content domain of quality of life for cancer patients with pain. Cancer Nursing, 13(2), 108-115.

Phillips, J.R. (1995). Quality of life research: Its increasing importance. Nursing Science Quarterly, 8(3), 100-101.

Osoba, D. (1994). Lessons learned from measuring health-related quality of life in oncology. Journal of Clinical Oncology, 12(3), 608-616.

Rosenberg, R. (1995). Health-related quality of life between naturalism and hermeneutics. Social Science \& Medicine, 41(10), 1411-1415.

Schmitt, R. (1993). Quality of life issues in lung cancer: New symptom management strategies. Chest, 103(1), 51S-55S.

Strauss, A.L., Corbin, J., Fagerhaugh, S., Glaser, B., Maines, D., Suczek, B., \& Weiner, C.L. (1984). Chronic illness and the quality of life (2nd ed.). St. Louis: Mosby.

Wyatt, G., Kurtz, M.E. \& Liken, M. (1993). Breast cancer survivors: An exploration of quality of life issues. Cancer Nursing, 16(6), 440-448.

Yasko, J.M. \& Fleck, A. (1984/1995). Health care reform and cost containment in oncology care. Oncology Nursing Forum, 22(3), 491-502. [first published in 1984].

Zacharias, D.R., Gilg, C.A. \& Foxall, M.J. (1994). Quality of life and coping in patients with gynecological cancer and their spouses. Oncology Nursing Forum, 21(10), 1699-1706. 